\title{
Singular Spectrum Analysis of Atrial Activations to Predict Atrial Fibrillation Recurrence after Ablation Procedure
}

\author{
Raquel Cervigón ${ }^{1}$, Javier Moreno ${ }^{2}$, José Millet ${ }^{3}$ and Francisco Castells ${ }^{3}$ \\ ${ }^{1}$ Escuela Politécnica de Cuenca, UCLM, Spain \\ ${ }^{2}$ Unidad de Arritmias, Hospital Ramón y Cajal, Madrid, Spain \\ ${ }^{3}$ ITACA, Universidad Politécnica de Valencia, Valencia, Spain
}

\begin{abstract}
The goal of pulmonary vein ablation for atrial fibrillation $(A F)$ is returning to normal sinus rhythm; nevertheless it success is limited in part by uncertainly in the mechanisms that sustain AF. $43 \mathrm{AF}$ patients were submitted to an ablation procedure and monitored after ablation. Dominant frequency from intra-atrial recordings obtained before the intervention were analysed in order to predict $A F$ recurrence. The novelty of this study is that dominant frequency was calculated from reconstructed signal by singular spectrum analysis (SSA) application. Patients that maintained sinus rhythm and patients with recurrence in $A F$ showed differences in left atrium dominant frequencies, with higher values in the recurrent $A F$ group than in group without recurrence in the arrhythmia $(p=0.02)$. Moreover, differences between both atria were found in the non-recurrent group, with $5.76 \pm 1.31 \mathrm{~Hz}$ in the left atrium vs. $6.25 \pm 1.23 \mathrm{~Hz}$ in the right atrium ( $p=0.03$ ). These findings show the potential of SSA as a preprocessing method and results are congruent with previous studies.
\end{abstract}

\section{Introduction}

Atrial fibrillation (AF) is the most frequent arrhythmia with higher prevalence in older persons[?,1]. Moreover, with the worldwide aging of the population an epidemic is forecasted within the next 10 to 20 years [3].

The electrophysiological mechanism involved is the presence of multiple simultaneous atrial wavefronts reentry circulating continuously and chaotically around the atria, whose foci in most cases are found in the pulmonary veins (PVs).

Treatment of AF is directed toward controlling underlying causes, slowing the heart rate and/or restore and maintain sinus rhythm to avoid the need for anticoagulation, reduce thromboembolic risk, prevent tachycardiainduced cardiomyopathy and improve survival. Pharmacology treatments have been commonly used for pa- tients suffering from this disease, nevertheless they are frequently ineffective and may be associated intolerable side effects [4].

The utilisation of catheter ablation as a potentially curative therapy for AF has been growing from the last decade. Using percutaneously introduced catheters, targeted areas of cardiac muscle are burned focally with special emphasis in the pulmonary veins.

Radiofrequency energy is applied to destroy this small area of tissue [5] to isolate pulmonary veins. The resulting tissue blocks the extra electrical signals from the pulmonary veins reaching the left atrium, so the area can no longer generate or conduct the fast, irregular impulses. This process is repeated around the opening of each of the four pulmonary veins. However, the long-term goal of this procedure is to eliminate the need for medications to prevent $\mathrm{AF}$.

Nevertheless, many aspects of the therapy are still controversial, patient management, prevention and treatment of complications and the most important, success rate, because at the moment it is difficult to predict when AF will be cured.

In this study we analyze the recurrence outcome of $\mathrm{AF}$ patients in three months follow-up, and we relate these results from singular spectrum analysis of atrial activations dominant frequency from both left and right atria (LA and RA, respectively).

\section{Materials}

$\mathrm{AF}$ intracardiac recordings were registered in 43 patients immediately before AF ablation procedure. Table 1 shows different parameters from recurrent and nonrecurrent $\mathrm{AF}$ patients where there was no statistically significant difference between groups. A 24-pole catheter (Orbiter, Bard Electrophysiology, 2-9-2 mm electrode spacing) was inserted through the femoral vein and positioned in the right atrium (RA) with the distal dipoles into the coronary sinus (CS) to record left atrial (LA) electrical 
activity as well. Twelve bipolar intracardiac electrograms were digitally recorded at $1 \mathrm{kHz}$ sampling rate (16 bit A/D conversion; Polygraph Prucka Cardio-Lab, General Electric). Fifty to 60 seconds recordings from paroxymal and persistent AF patients were analyzed. All patients were monitored after ablation, and were divided in 2 groups according to AF recurrence outcome 3 months after ablation procedure.

Table 1. Patient Clinical Characteristics

\begin{tabular}{lll}
\hline Parameters & Rec. AF & Non-rec. AF \\
\hline Paroxysmal AF Patients & 10 & 13 \\
Persistent AF Patients & 7 & 13 \\
Male (\%) & $11(65 \%)$ & $24(88 \%)$ \\
Age (years) & $51 \pm 14$ & $48 \pm 14$ \\
Structural heart disease (\%) & $4(29 \%)$ & $5(22 \%)$ \\
Left atrium size (mm) & $44 \pm 8$ & $44 \pm 6$ \\
\hline
\end{tabular}

\section{Methods}

\subsection{Singular Spectrum Analysis}

In recent years, a powerful technique such as Singular Spectrum Analysis (SSA) has been applied with great success in the field of time series analysis [6,7]. The possible application areas of SSA are diverse: from mathematics and non linear dynamics $[8]$ to medicine $[9,10]$.

SSA is a nonparametric method. It tries to overcome the problems of finite sample length and noisiness of sampled time series not by fitting an assumed model to the available series, but by using a data-adaptive basis set.

The basic SSA method consists of two complementary stages: decomposition and reconstruction; both stages include two separate steps. The main concept in studying the properties of SSA is 'separability', which characterizes how well different components can be separated from each other, in our case atrial activity and noise. Therefore, the absence of approximate separability is often observed in series with complex structure.

Basic SSA is performed in four steps. The first step, called the embedding step, transfers the one-dimensional time series $F=\left(f_{0}, \ldots, f_{N}\right)$ of length $N$, into a sequence of L-dimensional vectors $\mathbf{x}_{\mathbf{i}}=\left(\mathbf{f}_{\mathbf{i}}, \ldots, \mathbf{f}_{\mathbf{i}+\mathbf{L}-\mathbf{1}}\right)^{\mathbf{T}},(i=$ $1, \ldots, K) ; K=N-L+1$. The single parameter of this delay procedure is the window length $L,(1<L<N / 2)$ and longer than estimated time period. The $K$ vectors $\mathbf{x}_{\mathbf{i}}$ will form the columns of the $(L x K)$ trajectory matrix $\mathbf{X}$.

The next step is the Singular Value Decomposition (SVD) of the trajectory matrix into a sum of rank-one bi- orthogonal elementary matrices:

$$
\mathbf{X}=\mathbf{X}_{\mathbf{1}}+\ldots+\mathbf{X}_{\mathbf{L}}
$$

The elementary matrices $\mathbf{X}_{\mathbf{i}}$ are given by $\mathbf{X}_{\mathbf{i}}=$ $\mathbf{S}_{\mathrm{i}} \mathbf{U}_{\mathbf{i}} \mathbf{V}_{\mathbf{i}}^{\mathbf{T}}$, where $\mathbf{S}_{\mathbf{i}}$ matrix that contains the left-hand-side eigenvectors, $\mathbf{U}_{\mathbf{i}}$ represents the diagonal matrix that contains the singular values and $\mathbf{V}_{\mathbf{i}}$ is the $i$ th right-hand-side singular matrix of $\mathbf{X}$ (equivalent to the $i$ th eigenvector of $\left.\mathbf{X}^{\mathbf{T}} \mathbf{X}\right)$. The collection $\left(\mathbf{S}_{\mathbf{i}}, \mathbf{U}_{\mathbf{i}}, \mathbf{V}_{\mathbf{i}}\right)$ is called the $i$ th eigentriple of the SVD. These first two steps make up the decomposition stage of SSA and the next two steps the reconstruction stage.

In the third step for each group index, it is made a partition of the indices set $1, \ldots, L$ into $m$ disjoint subsets $I_{1}, \ldots, I_{m}$, corresponding to split the elementary matrices into $m$ groups. Let $\mathbf{X}_{\mathbf{I}_{\mathrm{m}}}=\mathbf{S}_{1} \mathbf{U}_{1} \mathbf{V}_{1}^{\mathbf{T}}$ represent the trajectory matrix for $I_{m}$. The resultant matrices are computed and substituting in equation 1 one obtains the new expansion

$$
\mathbf{X}_{\mathbf{I}}=\mathbf{X}_{\mathbf{I} 1}+\ldots+\mathbf{X}_{\mathbf{I m}}
$$

where the trajectory matrix is represented as a sum of $m$ resultant matrices. The last step in Basic SSA transforms each resultant matrix of the grouped decomposition (equation 2) into a new one dimensional series of length $N$, and is called diagonal averaging. Let $\mathbf{Y}$ be a $(L x K)$ matrix with elements $y_{i j}, 1 \leq i \leq L, 1 \leq j \leq K$. Make $L^{*}=\min (L, K), K^{*}=\max (L, K)$ and $N=L+K-1$. Let $y_{i j}^{*}=y_{i j}$ if $L<K$ and $y_{i j}^{*}=y_{j i}$ otherwise.

As a result, $\mathrm{N}$-sample sequences can be reconstructed from each grouped matrix in $\mathbf{I}_{\mathbf{i}}, 0 \leq i \leq m$ through diagonal average. Therefore, through the four steps in SSA, the original signal can be decomposed into $m$ components.

In our study, this method was applied to extract the main atrial activitiy information from electrograms from LA and RA were after a SSA transform uses the eigenvalues with A the length of the window correlated with the lag of between maximum autocorrelation [11].

\subsection{Frequency Analysis}

Power spectral density estimation is a commonly-used analytic technique for describing periodicities in time series, in this case to estimate the atrial activation rate. Firstly, main frequency was obtained according to the steps proposed by Botteron $[12,13]$ and the second way it was to apply SSA, reconstructing the analyzed signals from the main principal components.

The main or dominant frequency (DF) was identified as the frequency relating to the maximum amplitude in the frequency band between 3 and $10 \mathrm{~Hz}$. This is done independently for each of the five electrograms obtained from the catheter. The mean DF value is obtained as the representative DF value for each $8 \mathrm{~s}$ segment. 


\subsection{Statistical Analysis}

As a final step statistical techniques were applied to the extracted parameters. The normal distribution of the variables was firstly validated by the Shapiro-Wilk test and subsequently Student's unpaired t -test was carried out to check the difference between parameters derived from both groups. The parameters were expressed in terms of mean and standard deviation values. Results were considered to be statistically significant at $p<0.05$.

\section{Results}

SSA preprocessing was superior to the method proposed by Botteron when the signal was very irregular. Neverheless, it also works in more regular pattern. Moreover, atrial activity DF extracted from both methods show a high correlation. (Figure 1).

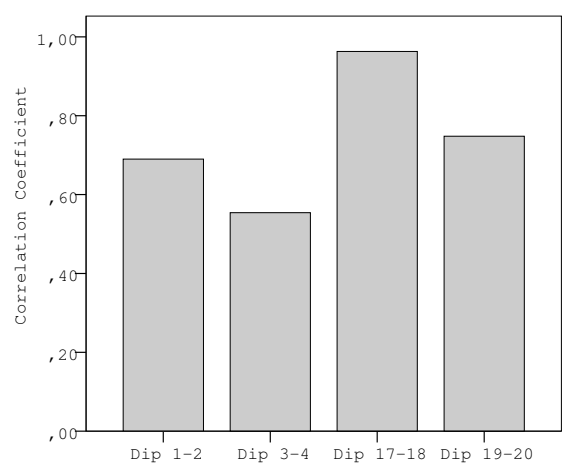

Figure 1. Correlation coefficient between dominant frequency from SSA reconstructed signals and Botteron preprocessing

Atrial activity DF were studied in order to predict the outcome of the procedure. Several analysis on different atrial chambers were performed separately. DF from patients without recurrences in the arrhythmia showed differences between both atria $(\mathrm{p}=0.03)$, with $5.76 \pm 1.31 \mathrm{~Hz}$ in the LA and $6.25 \pm 1.23 \mathrm{~Hz}$ in the RA, nevertheless DF from patients with AF recurrence showed similar values of DF between both atrium, without statistically significant differences between both atria, with $6.83 \pm 1.07 \mathrm{~Hz}$ in the $\mathrm{LA}$ and $6.38 \pm 1.00 \mathrm{~Hz}$ in the RA. Moreover, differences in DF along the LA showed differences between patients with recurrences in the arrhythmia $(\mathrm{p}=0.02), 6.61 \pm 1.66$ $\mathrm{Hz}$ and those patients that maintenance sinus rhythm with $5.49 \pm 1.41 \mathrm{~Hz}$ (figure 2).

\section{Conclusions}

The search for predictors of AF ablation success and AF recurrence is currently of high clinical interest. On multi-

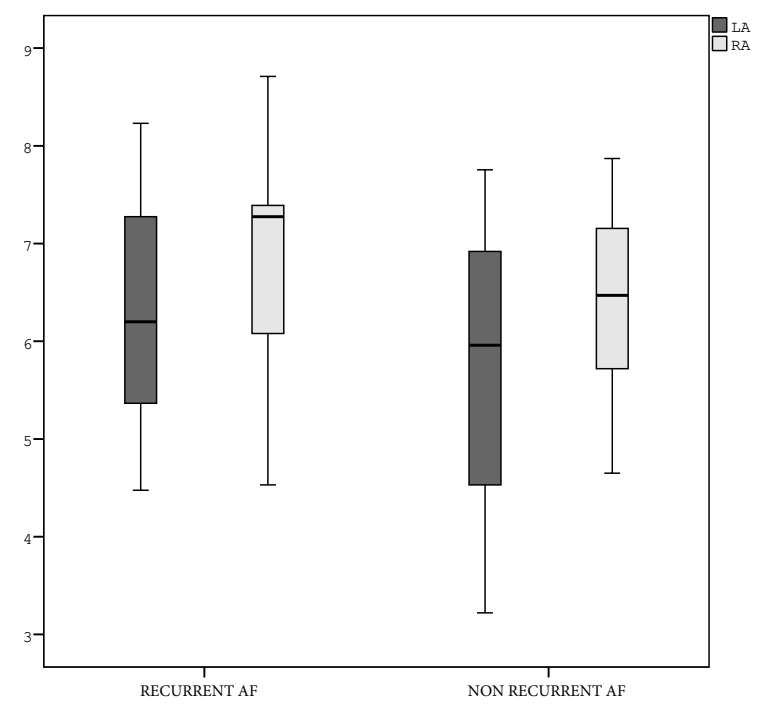

Figure 2. DF differences along LA and RA

variate analysis, the predictors of overall clinical success include: AF termination by ablation, a shorter ablation duration, younger age, male gender, and the presence of hypertension [14]. Moreover, the dominant atrial frequency has been described as a predictor of AF ablation outcome, where long cycle lengths were responsible for the organization of the arrhythmia, whereas the shortest cycle lengths were related to recurrences [15].

The high-DF sites produce a favourable condition for maintenance of AF [16]. Based on mechanistic studies, these areas exhibit higher dominant frequency [17], higher entropy [18] and lower causality from the pulmonary veins to the atria [19], distinguishing them from other atrial sites [20].

Therefore, DF is a key parameter for the analysis of AF. In this work we propose SSA, as a new preprocessing approach to estimate the dominant atrial rate. In the area of nonlinear time series analysis SSA was considered as a technique that could compete with more standard methods. It provides information about the deterministic and stochastic parts of the time series even when the series is short and noisy, very useful in AF recordings. The results of this study illustrate that SSA was able to extract main components of atrial activity time series with characteristic irregular behaviour, showing a high correlation with previous preprocessing methods.

The results of DF measurements obtained in this study were analyzed according to atrial chamber. DF values exhibited a RA to LA gradient, with higher values at the LA in patients that maintenance sinus rhythm, nevertheless this gradient is not shown in the patients with recurrences in the arrhythmia. Furthermore, numerical results show that patients patients within the recurrent AF group 
had higher DF values. This means, in turn, that patients with a more quickly atrial electrical activity will have a higher risk to AF recurrence.

As conclusion, DF estimation from reconstructed SSA intracardiac electrograms can prognosticate AF recurrences after successful ablation of pulmonary veins. High DF values in the LA, specially without a gradient between both atria, predict a very high-risk for AF recurrence. This suggests that when the atrial electrical activity is more rapid and similar in both atria, the reversion to sinus rhythm is more difficult. Similar results were obtained by non-linear morphology measurements [21]. These findings show that DF measurements mediated by SSA can be helpful as an indicator of risk for AF recurrence after successful ablation of AF.

\section{References}

[1] Chugh SS, Havmoeller R, Narayanan K, Singh D, Rienstra M, Benjamin EJ, Gillum RF, Kim YH, McAnulty Jr JH, Zheng ZJ, Forouzanfar MH, Naghavi M, Mensah GA, Ezzati M, Murray CJL. Worldwide epidemiology of atrial fibrillation: a global burden of disease 2010 study. Circulation Feb 2014;129(8):837-47.

[2] Kirchhof P, Benussi S, Kotecha Dea. 2016 esc guidelines for the management of atrial fibrillation developed in collaboration with eacts. Rev Esp Cardiol Engl Ed Jan 2017; 70(1):50.

[3] Morillo CA, Banerjee A, Perel P, Wood D, Jouven X. Atrial fibrillation: the current epidemic. J Geriatr Cardiol Mar 2017;14(3):195-203.

[4] Al-Khatib SM, Arshad A, Balk EM, Das SR, Hsu JC, Joglar JA, Page RL. Risk stratification for arrhythmic events in patients with asymptomatic pre-excitation: A systematic review for the $2015 \mathrm{acc} / \mathrm{aha} / \mathrm{hrs}$ guideline for the management of adult patients with supraventricular tachycardia: A report of the american college of cardiology/american heart association task force on clinical practice guidelines and the heart rhythm society. Circulation Apr 2016;133(14):e57586.

[5] Haissaguerre M, Shah D, Jais P. Electrophysiological breakthroughs from the left atrium to the pulmonary veins. Circulation 2000;102:2463ñ5.

[6] Elsner JB, Tsonis AA. Singular spectrum analysis: a new tool in time series analysis. New York: Plenum Press, 1996. ISBN 0306454726.

[7] Golyandina N, Nekrutkin VV, Zhigliavskii AA. Analysis of time series structure: SSA and related techniques, volume 90. Boca Raton, Fla.: Chapman Hall/CRC, 2001. ISBN 1584881941 (acid-free paper).

[8] Kember G, Flower AC, Holubeshen J. Forecasting river flow using nonlinear dynamics 1993;7(3):205-212.

[9] Mamou J, Feleppa EJ. Singular spectrum analysis applied to ultrasonic detection and imaging of brachytherapy seeds. The Journal of the Acoustical Society of America 03 2007; 121(3):1790-1801.
[10] Warrick PA, Precup D, Hamilton EF, Kearney RE. Fetal heart rate deceleration detection using a discrete cosine transform implementation of singular spectrum analysis. Methods Inf Med 2007;46(2):196-201.

[11] Vautard R, Ghil M. Singular spectrum analysis in nonlinear dynamics, with applications to paleoclimatic time series. Physica D Nonlinear Phenomena 1989;35(3):395 424. ISSN 0167-2789.

[12] Botteron G, Smith J. A technique for measurements of the extent of spatial organization of atrial activation during atrial fibrillation in the intact human heart. IEEE Trans Biomed Eng 1995;42:579-586.

[13] Castells F, Cervigón R, Millet J. On the preprocessing of atrial electrograms in atrial fibrillation: Understanding botteron's approach. Pacing Clin Electrophysiol Nov 2013;

[14] Heist EK, Chalhoub F, Barrett C, Danik S, Ruskin JN, Mansour M. Predictors of atrial fibrillation termination and clinical success of catheter ablation of persistent atrial fibrillation. Am J Cardiol Aug 2012;110(4):545-51.

[15] Okumura Y, Watanabe I, Kofune M, Nagashima K, Sonoda K, Mano H, Ohkubo K, Nakai T, Hirayama A. Characteristics and distribution of complex fractionated atrial electrograms and the dominant frequency during atrial fibrillation: relationship to the response and outcome of circumferential pulmonary vein isolation. J Interv Card Electrophysiol Sep 2012;34(3):267-75.

[16] Narayan SM, Krummen DE, Rappel WJ. Clinical mapping approach to diagnose electrical rotors and focal impulse sources for human atrial fibrillation. J Cardiovasc Electrophysiol May 2012;23(5):447-54.

[17] Atienza F, Almendral J, Jalife J, Zlochiver S, Ploutz-Snyder R, Torrecilla EG, Arenal A, Kalifa J, Fernández-Avilés $\mathrm{F}$, Berenfeld O. Real-time dominant frequency mapping and ablation of dominant frequency sites in atrial fibrillation with left-to-right frequency gradients predicts longterm maintenance of sinus rhythm. Heart Rhythm Jan 2009; 6(1):33-40.

[18] Cervigón R, Moreno J, García-Quintanilla J, PérezVillacastín J, Castells F. Entropy at the right atrium as a predictor of atrial fibrillation recurrence outcome after pulmonary vein ablation. Biomed Tech Berl Jun 2015;

[19] Cervigón R, Castells F, Gómez-Pulido J, Pérez-Villacastín J, Moreno J. Granger causality and jensen-shannon divergence to determine dominant atrial area in atrial fibrillation. Entropy 2018;20(1):57.

[20] Jalife J. Spatial and temporal organization in ventricular fibrillation. Trends Cardiovasc Med Jul 1999;9(5):119-27.

[21] Cervigón R, Moreno J, Castells F. Entropy analysis of atrial activity morphology to study atrial fibrillation recurrences after ablation procedure. In Bioinformatics and Biomedical Engineering - Third International Conference, IWBBIO 2015, Granada, Spain, April 15-17, 2015. Proceedings, Part I. $2015 ; 146-154$.

Address for correspondence:

Raquel Cervigón Abad

Escuela Polictécnica. Camino del Pozuelo sn. Cuenca 16071.

raquel.cervigon@uclm.es 